\title{
Indicadores Cognitivos, Lingüísticos, Comportamentais e Acadêmicos de Pré-Escolares Nascidos Pré-Termo e a Termo ${ }^{1}$
}

\author{
Christyne Gomes Toledo de Oliveira ${ }^{2}$ \\ Sônia Regina Fiorim Enumo \\ Sávio Silveira de Queiroz \\ Universidade Federal do Espírito Santo \\ Romildo Rocha Azevedo Jr \\ Centro Universitário de Vila Velha
}

\begin{abstract}
RESUMO - Este estudo comparou o desenvolvimento de crianças, aos cinco anos de idade, nascidas prematuras e com baixo peso (PTBP), com crianças nascidas a termo (AT), avaliando-se indicadores acadêmicos (IAR), comportamentais (CBCL-11/25 anos), linguísticos (LAVE-expressiva; TVIP-receptiva) e cognitivos (testes psicométricos: Columbia e Raven-MPC; prova assistida: CATM). A amostra foi composta por 34 crianças distribuídas em dois grupos: G1-PTBP: 17 crianças pré-termo ( $<37$ semanas de gestação) e baixo peso; e G2-AT: 17 crianças a termo e peso $\geq 2.500 \mathrm{~g}$. G1-PTBP apresentou desempenho inferior nas áreas acadêmica, lingüística expressiva, comportamental e cognitiva (Columbia e CATM: operações cognitivas e comportamentos facilitadores). Confirmaram-se outros estudos indicativos de que crianças PTBP podem apresentar mais problemas no curso de seu desenvolvimento do que crianças nascidas a termo.
\end{abstract}

Palavras-chaves: prematuridade; desempenho cognitivo; fatores de risco ao desenvolvimento.

\section{Cognitive, Linguistic, Behavioral and Academic Indicators of Preterm and Full Term Born Preschool Children}

\begin{abstract}
This study compared the development of 5 years old premature and low weight born children, with full term born children of the same age. The following indicators were evaluated: academic (IAR), behavioral (CBCL-1 1/2-5 years), language (LAVE-expressive, TVIP-receptive) and cognitive (Columbia, Raven-MPC and CATM). The sample consisted of 34 children: 17 premature and low weight born children ( $<37$ weeks' gestation), and 17 full-term born children who weighed $\geq 2.500 \mathrm{~g}$ as newborns. The premature children showed a lower performance on academic, expressive language, behavioral and cognitive areas (Columbia and CATM: cognitive operations and facilitating behaviors). The study confirmed other studies indicating that premature children can present more problems in the course of their development than full term born children.
\end{abstract}

Keywords: prematurity, cognitive performance; development risk factors.

Os avanços na área da Neonatologia tem promovido um aumento na sobrevivência de recém-nascidos com idade gestacional e peso de nascimento menores (Rugolo, 2005). Entretanto, os bebês que nascem prematuros (PT) (antes de 37 semanas de gestação) e com baixo peso $(\mathrm{BP})(<2.500 \mathrm{~g})$ são mais vulneráveis a doenças e complicações, como enfermidades crônicas (broncodisplasia, síndrome do estresse respiratório, hemorragia intraventricular, retinopatia ou anemia da prematuridade, problemas cardiovasculares, dentre outros) e hospitalizações repetidas e/ou prolongadas (Bordin, Linhares \& Jorge, 2001; Klebanov \& Brooks-Gunn, 2006; Linhares, Chimello, Bordin, Carvalho \& Martinez, 2005).

Diante disso, de acordo com Bordin et al. (2001), a busca de entendimento sobre o impacto do nascimento prematuro no crescimento e desenvolvimento de crianças, em diferentes idades, tem motivado várias pesquisas, sobretudo na área da Psicologia. Tais pesquisas apontam que crianças PTBP, em comparação com crianças nascidas a termo (AT), são

1 Apoio: $\mathrm{CNPq}$

2 Endereço para correspondência: Rua Júlia Lacourt Penna, 415, Apto. 405, Jardim Camburi. Vitória, ES - CEP 29090-210. E-mail: cgtoliveira@gmail.com mais propensas a apresentar alterações no desenvolvimento cognitivo, acadêmico, comportamental e da linguagem. Mais especificamente, essas crianças apresentam mais dificuldades na linguagem receptiva e na expressiva (Pedromônico, 2006; Pereira \& Funayama, 2004) e nas áreas de coordenação visomotora, planejamento e organização, formação de conceitos verbais e numéricos, pensamento racional e associativo, capacidade de síntese, orientação espacial e memória remota, ao mesmo tempo em que apresentam maior incidência de problemas de aprendizagem, de Transtorno de Déficit de Atenção/Hiperatividade e outras desordens comportamentais e psiquiátricas, com destaque para problemas internalizantes e externalizantes, com alta reatividade infantil ou passividade, emocionalidade e afetividade negativa, que afetam a interação mãe-criança e as relações familiares (Bhutta, Cleves, Casey, Cradock \& Anand, 2002; Bohnert \& Breslau, 2008; Feldman, 2007; Linhares et al., 2005; Martins, Linhares \& Martinez, 2005; Méio et al., 2004).

Essas áreas do desenvolvimento infantil costumam ser avaliadas por instrumentos psicométricos tradicionais, nos quais é identificado um baixo desempenho de crianças em risco para problemas de desenvolvimento. Assim, considera-se 
importante adotar uma avaliação psicológica que identifique, além das dificuldades, os recursos potenciais dessas crianças, a exemplo da avaliação assistida (Linhares, Escolano \& Enumo, 2006). Segundo Tzuriel (2001), a avaliação assistida é uma avaliação do pensamento, percepção, aprendizado e resolução de problemas, para um ativo processo de ensino direcionado à modificação do funcionamento cognitivo. É, portanto, uma avaliação dinâmica e interativa, na qual há a assistência do examinador, que fornece suporte instrucional ao examinado durante o processo de avaliação, possibilitando que a criança revele seu desempenho potencial, as estratégias que usa para aprender e sua sensibilidade à instrução (Enumo, 2005). Tem sido usada de forma complementar à avaliação tradicional, que informa o nível de desempenho intelectual da criança. Assim, avaliação cognitiva não deve basear-se em um único teste, sendo indicada uma combinação de procedimentos de diferentes abordagens, no intuito de melhor compreender o funcionamento cognitivo da criança (Haywood \& Lidz, 2007).

Nesse contexto de avaliação, destaca-se, aqui, a importância de avaliar diferentes dimensões do contexto e do comportamento das crianças em risco para problemas de desenvolvimento, a fim de fornecer subsídios para intervenções capazes de minimizar as dificuldades presentes nas suas trajetórias de desenvolvimento (Linhares, Carvalho, Correia, Gaspardo \& Padovani, 2006; Luthar, Sawyer \& Brown, 2006; Olson, Sameroff, Lunkenheimer \& Kerr, 2009).

Com esse enfoque, este estudo tem como objetivo avaliar o desempenho cognitivo, por uma combinação de procedimentos psicométricos e assistido, e os indicadores comportamentais, acadêmicos e lingüísticos de crianças nascidas prematuras e com baixo peso, aos cinco anos de idade, comparadas com crianças nascidas a termo.

\section{Método}

\section{Participantes}

A amostra foi composta por 34 crianças, com idade entre cinco anos e cinco anos e 11 meses, de ambos os sexos (18 meninos), subdivididas em dois grupos:

1) G1-PTBP - 17 nascidas pré-termo (gestação $<37$ semanas) e com baixo peso $(<2.500 \mathrm{~g})$, inscritas no Serviço de Follow-up do Ambulatório de Neurologia de um hospital público de uma cidade metropolitana da região Sudeste, por problemas no nascimento decorrentes da prematuridade e do baixo peso, frequentando a pré-escola (Pré-II) de escolas públicas de Educação Infantil. Essa amostra foi extraída de 81 crianças dessa faixa etária, da qual foram excluídas 64 crianças por erro nos telefones ou endereços (52), pela ausência destes (5), pelos pais se recusarem a participar por residir longe do hospital (5) ou em outro Estado (2);

2) G2-AT - 17 crianças nascidas a termo e peso $\geq 2.500 \mathrm{~g}$, matriculadas na classe de pré-escola (Pré-II) de uma escola pública de Educação Infantil, localizada em um bairro próximo ao hospital.

No G1-PTBP, 12 famílias tinham nível socioeconômico da classe $\mathrm{C}$ e cinco da classe B; no G2-AT, 15 eram da classe C e duas da classe B. Assim, a maioria da amostra estava na classe $\mathrm{C}$, que corresponde a um ganho mensal entre $1 \frac{1}{2}$ e três salários-mínimos da época (Associação Brasileira de Empresas de Pesquisa -ABEP, 2008), mostrando que os grupos eram equivalentes socialmente.

\section{Instrumentos}

Para a coleta de dados, foram utilizados 11 instrumentos, relacionados às áreas:

1) acadêmica: Instrumento de Avaliação do Repertório Básico para Alfabetização - IAR (Leite, 1984): 13 tarefas que avaliam habilidades e conceitos básicos para a alfabetização no país, para as quais a criança deve emitir respostas escritas ou verbais;

2) comportamental: Child Behavior Checklist (CBCL 1 1/2-5 anos) (Achenbach \& Rescorla, 2004), os pais respondem uma escala de comportamentos relacionados à competência social, depressão e isolamento, agressividade, comportamento antissocial e ansiedade, comparando os comportamentos do filho com os de outra criança ou classificando-o em relação à freqüência em que ocorrem. Tem sido um dos instrumentos mais utilizado para a avaliação da saúde mental de crianças e adolescentes no mundo (Gauy \& Guimarães, 2006), com validação parcial em nosso país (Bordin, Mari \& Caieiro, 1995);

3) linguística: (a) Teste de Vocabulário por Imagens Peabody - TVIP (Capovilla \& Capovilla, 1997) - avalia o desenvolvimento lexical no domínio receptivo (compreensão de vocabulário), de crianças e jovens entre 2 anos e 6 meses até 18 anos de idade; (b) Lista de Avaliação do Vocabulário Expressivo - LAVE (Capovilla \& Capovilla, 1997) - avalia o vocabulário expressivo de crianças entre dois e seis anos de idade, com normas para a população brasileira;

4) cognitiva - (a) provas psicométricas: Escala Columbia de Maturidade Intelectual (Burgmeister, Blum \& Lorge, 2001) - fornece uma estimativa de aptidão geral de raciocínio e habilidade de classificação de crianças entre 5 e 9 anos; e Matrizes Progressivas Coloridas de Raven - Raven-MPC (Angelini, Alves, Custódio, Duarte \& Duarte, 1999) - teste de inteligência não-verbal que avalia o raciocínio analógico-dedutivo de crianças de 5 a 11 anos; ambas com padronização nacional;

(b) prova assistida, em que o sujeito funciona como seu próprio controle, com medidas antes e após a mediação do aplicador: Children's Analogical Thinking ModifiabilityCATM (Tzuriel \& Klein, 1990) adaptada por Santa Maria e Linhares (1999) - avalia o raciocínio analógico-dedutivo (A:B:C:?), no formato não-verbal. É composta por 32 itens, em ordem crescente de dificuldade, divididos em cinco fases: preliminar, sem ajuda (SAJ), assistência (ASS) (com vários níveis estruturados de ajuda da mediadora), manutenção (MAN) e transferência (TRF). Tem sido usado em pesquisas nacionais há mais de 10 anos (Santa Maria \& Linhares, 1999; Turrini, Enumo, Ferrão \& Monteiro, 2010, por exemplo).

Além dessas provas, foram utilizados 3 protocolos de registro de comportamentos e de operações cognitivas: (a) Operações cognitivas facilitadoras e não-facilitadoras e (b) Comportamentos facilitadores e não-facilitadores (Linhares, Escolano \& Enumo, 2006); e (c) Comportamentos afetivo- 
-motivacionais - Behavior Observation Rating Scale (BORS), contida na ACFS (Applications of Cognitive Functions Scale) (Lidz \& Jepsen, conforme citado por Haywood \& Lidz, 2007), já utilizada no país (Turrini et al., 2010).

Para obtenção de informações gerais sobre a criança e sua família, como a história pessoal (gestação, infância, escolaridade), hábitos e interesses, deficiência, história familiar, história sócioeconômica, características comportamentais, cognitivas e emocionais da criança, foi utilizado o protocolo de entrevista de Anamnese (Carretoni Filho \& Prebianchi, 1994). Para a seleção da amostra e caracterização dos dados pessoais e neonatais (nome, idade, endereço, telefone, peso e idade gestacional) das crianças do G1-PTBP, foram utilizados os prontuários de acompanhamento do Serviço de Follow-up do hospital, e para G2-AT, o Caderno de Registro de Matrícula Escolar, que continha o Cartão de Vacina, com os dados de nascimento.

A avaliação do nível sócio-econômico das famílias foi feita pelo Critério de Classificação Econômica Brasil (ABEP, 2008).

\section{Procedimento}

A pesquisa foi realizada em três etapas, descritas a seguir:

Etapa 1 - Identificação e caracterização dos participantes: para G1-PTBP, utilizou-se os prontuários de acompanhamento do Serviço de Follow-up do hospital e, para G2-AT, o Caderno de Registro de Matrícula Escolar. Após a identificação das crianças de ambos os grupos, foram feitos contatos via telefone e carta com os responsáveis, convidando-os a participar da pesquisa. Em caso afirmativo, marcava-se um horário para leitura, assinatura do termo de consentimento e início das avaliações.

Etapa 2 - Coleta de dados com os responsáveis: as mães responderam à entrevista de anamnese, o protocolo de avaliação do nível sócio-econômico (ABEP, 2008), o instrumento de avaliação da linguagem expressiva das crianças (LAVE) e de problemas de comportamento da criança (CBCL - 11/2 a 5 anos);

Etapa 3 - Coleta de dados com as crianças identificadas previamente pela pesquisadora: foi realizada individualmente em quatro sessões, com intervalo semanal, nas quais foram aplicados: (a) o IAR, com a duração de 40 min; (b) a Escala Columbia e o TVIP, com a duração de 45 min; (c) três fases do CATM: SAJ (sem ajuda do examinador), ASS (com assistência do examinador) e MAN (sem ajuda do examinador), com duração de 45 min; (d) a fase TRF do CATM e o Raven, com a duração de $45 \mathrm{~min}$. As aplicações do CATM foram gravadas em vídeo para análise posterior dos dados.

A coleta dos dados com a criança e com os pais foi feita concomitantemente $^{3}$, sendo realizada no hospital com G1-PTBP e na escola pública com G2-AT, após aprovação do Comitê de Ética do hospital e autorização dos responsáveis pelos serviços do hospital e da escola, e dos pais.

Posteriormente à fase de coleta de dados, as crianças do G1-PTBP participaram de um programa de educação cognitiva como parte de uma tese de Doutorado ${ }^{4}$.

3 Toda a coleta de dados foi conduzida pela pesquisadora, que tem formação em Psicologia, com auxílio de uma aluna de doutorado em Psicologia e duas bolsistas de Iniciação Científica.

4 Turrini, A. F. (2011). Comportamentos afetivo-motivacionais e de

\section{Processamento e análise dos dados}

Os instrumentos foram assim processados: (a) instrumentos psicométricos: a correção e análise dos testes IAR, LAVE, TVIP, Raven-MPC, Columbia, e CBCL ( $1 / 2$ a cinco anos), seguiram os critérios dos respectivos manuais; (b) prova assistida: Children's Analogical Thinking Modifiability - CATM (Tzuriel \& Klein, 1990) - foram feitas comparações das proporções de acertos em cada fase (sem ajuda- SAJ, assistência- ASS, manutenção- MAN e transferência- TRF) (Santa Maria \& Linhares, 1999). A partir desses dados, obtém-se os perfis de desempenho cognitivo ${ }^{5}$ : alto-escore, ganhador, não-mantenedor, transferidor e não-transferidor. Para comparação entre os dois grupos, utilizou-se a proporção de acerto total na MAN, que avalia a manutenção das estratégias de resolução de problemas após a suspensão da ajuda da examinadora (Linhares, Escolano \& Emuno, 2006).

Também foram analisados os seguintes dados do CATM: (a) Níveis de ajuda oferecidos pela examinadora na fase de assistência, que vão desde a Repetição da Instrução (nível 1) até à demonstração da resolução de um arranjo pelo examinador (nível 4) (Santa Maria \& Linhares, 1999). Para cada cartão, foi calculado o nível de ajuda fornecido e a média de ajuda na fase ASS, para cada grupo; (b) Operações cognitivas facilitadoras e não-facilitadoras (Linhares, Santa Maria \& Escolano, 2006) - cada operação cognitiva facilitadora recebeu 1 ponto e sua oposta, não-facilitadora, recebeu pontuação 0 , calculando-se a proporção de operações facilitadoras de cada criança e a média dos grupos; (c) Comportamentos facilitadores e não-facilitadores (Linhares, Santa Maria \& Escolano, 2006) - cada comportamento facilitador recebeu um ponto e seu oposto, não-facilitador, recebeu pontuação 0 , calculando-se a proporção de comportamentos facilitadores de cada criança e a média dos grupos; (d) Comportamentos afetivo-motivacionais (Applications of Cognitive Functions Scale-ACFS- Lidz \& Jepsen, conforme citado por Haywood \& Lidz, 2007) - cada comportamento desta escala foi pontuado em: 0 (não há evidência), 1 (ocorrência inconsistente) ou 2 (ocorrência ótima), obtendo-se o máximo de 8 pontos por comportamento e de 14 pontos por fase. Após, foi calculada a pontuação média de cada comportamento por criança e a média de cada grupo.

Para garantir a fidedignidade da avaliação dos protocolos de operações cognitivas, de comportamentos e da ACFS, foram sorteados seis protocolos $(20 \%)$, sendo três de cada

autorregulação em pré-escolares nascidos prematuros e com baixo peso: avaliação e intervenção em estratégias metacognitivas. Tese de Doutorado. Programa de Pós-Graduação em Psicologia, Universidade Federal do Espírito Santo. Vitória, ES.

5 Perfis de desempenho cognitivo- alto-escore: a criança apresenta soluções analógicas eficientes na fase inicial sem ajuda (SAJ) e, no mínimo, 0,60 de acertos na fase de manutenção (MAN); ganhador: melhora o desempenho na fase de assistência (ASS) e o mantém na proporção de, pelo menos, 0,50 de acertos após a suspensão da ajuda da examinadora; não-mantenedor: não melhora o seu desempenho na fase ASS, comparado à SAJ ou apresenta uma melhora em proporção abaixo de 0,50 de acertos na ASS, mas isto não se mantém na MAN transferidor: a criança apresenta um bom desempenho na fase de transferência, na proporção de 0,60 de acertos; não-transferidor: a criança apresenta menos de 0,60 de acertos na fase de transferência (Linhares, Santa Maria \& Escolano, 2006). 
Tabela 1. Comparações dos indicadores acadêmicos, lingüísticos e comportamentais de pré-escolares nascidos prematuros e com baixo peso (G1) e nascidos a termo (G2)

\begin{tabular}{|c|c|c|c|c|c|}
\hline \multirow[b]{2}{*}{ Provas } & \multicolumn{2}{|c|}{ G1-PTBP } & \multicolumn{2}{|c|}{ G2-AT } & \multirow{2}{*}{$\begin{array}{l}\text { G1 X G2 } \\
\text { (p-valor) }\end{array}$} \\
\hline & M & DP & M & DP & \\
\hline IAR - Escore Total & 0,71 & 0,11 & 0,77 & 0,06 & $0,032 *$ \\
\hline TVIP - Escore Bruto & 53,94 & 6,99 & 56,71 & 5,85 & 0,222 \\
\hline LAVE - Escore Bruto & 277,35 & 26,99 & 296,71 & 7,03 & $0,011^{*}$ \\
\hline CBCL -Total de problemas & 59,06 & 11,68 & 52,82 & 6,12 & 0,060 \\
\hline
\end{tabular}

Legenda: $\mathrm{M}=$ média; $\mathrm{DP}=$ desvio-padrão; * $p$-valor $\leq 0,05$ indica significância pelo teste de $t$ de Student.

grupo. $\mathrm{O}$ cálculo de concordância foi feito entre três juizes (AB, BC e AC), adotando-se 70\% como índice mínimo de concordância (Fagundes, 1982). Foram obtidos índices de concordância de $79 \%$ para as operações cognitivas, e de $86 \%$ para os comportamentos registrados.

Após a análise descritiva dos dados, foram feitas análises estatísticas inferenciais, com nível de significância de 0,05. Para verificar se as diferenças entre os grupos (PTBP vs. AT) tinham significância estatística, foram comparados, pelo teste t de Student, os dados referentes aos indicadores de desempenho nas provas cognitivas (percentil no Raven-MPC e Columbia, proporção de acertos totais na fase da MAN no CATM, proporção de operações cognitivas e comportamentos facilitadores nas fases do CATM, proporção dos níveis de ajuda utilizados na fase ASS do CATM), lingüísticas (escore no TVIP e LAVE), acadêmicas (proporção de acertos Total no IAR) e comportamental (escore no CBCL) (Pestana \& Gageiro, 2003). Para o tratamento estatístico dos dados, utilizou-se o Statistical Package for Social Sciences (SPSS)® na versão 15.0 .

\section{Resultados}

Os grupos foram pareados quanto à variável sexo $(53 \%$ de meninos) e à variável idade ( $\mathrm{Md}=5$ anos e 6 meses $)$. Para G1-PTBP, a mediana de peso ao nascimento foi de 1.895 gramas e da idade gestacional foi de 34,2 semanas, enquanto, para G2-AT, foi de 3.175 gramas e 40 semanas, respectivamente. $\mathrm{Na}$ época do parto, a mediana de idade das mães das crianças do G1-PTBP foi de 28 anos (AV=18-41) e do G2-AT, 26 anos (AV=16-41). A mediana de escolaridade das mães do G1-PTBP foi de nove anos (AV = 4-11), equivalendo ao Ensino Fundamental completo, e a do G2-AT foi de sete anos $(\mathrm{AV}=0-11)$, correspondente à $6^{\mathrm{a}}$ série do Ensino Fundamental, sendo que uma das mães nunca freqüentou o ensino regular, mas sabia ler e escrever.

\section{Avaliação acadêmica, da linguagem e comportamental}

Em relação ao repertório acadêmico das crianças, pode-se verificar na Tabela 1, que G1-PTBP apresentou desempenho significativamente menor do que G2-AT, demonstrando mais dificuldade nas habilidades básicas para a aprendizagem da leitura e escrita.

$\mathrm{Na}$ avaliação da linguagem, houve diferença estatisticamente significativa entre os grupos no vocabulário expressivo pela LAVE, em que G1-PTBP apresentou escore médio de 277 pontos, considerado abaixo da média para a idade de 5 anos $($ Média esperada $=296)($ Tabela 1$)$. Em contrapartida, G2-AT atingiu a média esperada, com 296 pontos. Assim, as crianças PTBP apresentaram mais dificuldades no repertório verbal, com média de palavras faladas abaixo da esperada para idade.

Adicionalmente aos resultados da Tabela 1, a análise dos dados da avaliação da linguagem receptiva (TVIP) mostrou que não houve diferença estatisticamente significativa entre os grupos $[\mathrm{G} 1-\mathrm{PTBP}=53,94( \pm 6,99)$; G2-AT $=56,71$ $( \pm 5,85) ; p \leq 0,22]$.

De acordo com a percepção das mães quanto à avaliação de problemas de comportamentos apresentados pelo filho (CBCL 1 1 1/2-5 anos), verifica-se que a média de T score de ambos os grupos, na Escala Total de Problemas, ficou dentro do intervalo menor do que $65(\mathrm{AV}=53-63)$, ou seja, enquadra-se na categoria Não-Clínica e não houve diferença estatística entre os grupos (Tabela 1).

Analisando os dados do CBCL (11/2-5 anos) segundo os critérios de classificação do DSM-IV, obteve-se uma diferença significativa entre os grupos nas escalas de Transtornos de Ansiedade, que incluem sintomas, como não esperar, não tolerar as coisas for do lugar, ficar perturbada com alguma mudança na rotina, e na escala de Problemas Desenvolvimentais Invasivos, que incluem problemas do tipo: choro imotivado, irritabilidade e isolamento social (Tabela 2).

\section{Avaliação cognitiva}

Na avaliação cognitiva psicométrica, houve diferença estatisticamente significativa entre os grupos, na Escala Columbia. Embora os percentis de ambos os grupos esteja dentro da média, de acordo com as normas da Escala, a média de acertos do G1-PTBP foi significativamente mais baixa (Tabela 3 ). Essas crianças apresentaram índices mais baixos de raciocínio geral envolvendo a habilidade de classificação (por sexo, idade, partes do corpo, número, por exemplo), em comparação às crianças nascidas a termo. Em contrapartida, a análise dos dados do Raven-MPC mos- 
Tabela 2. Comparação de indicadores de problemas de comportamento nas escalas do CBCL (11/2-5 anos) em pré-escolares nascidos prematuros e com baixo peso (G1) e a termo (G2)

\begin{tabular}{|c|c|c|c|c|c|c|c|c|c|}
\hline \multirow[b]{3}{*}{ Escalas do CBCL (DSM-IV) } & \multicolumn{4}{|c|}{ G1-PTBP } & \multicolumn{4}{|c|}{ G2-AT } & \multirow{3}{*}{$\begin{array}{l}\mathrm{G} 1 \mathrm{X} \mathrm{G} 2 \\
\text { ( } p \text {-valor) }\end{array}$} \\
\hline & \multicolumn{2}{|c|}{ Clínico } & \multirow{2}{*}{ Média } & \multirow{2}{*}{$\mathrm{DP}$} & \multicolumn{2}{|c|}{ Clínico } & \multirow{2}{*}{ Média } & \multirow{2}{*}{$\mathrm{DP}$} & \\
\hline & $f$ & $\%$ & & & $f$ & $\%$ & & & \\
\hline Transtornos afetivos & 4 & 23 & 59 & 10,9 & 2 & 11 & 56 & 6,16 & 0,753 \\
\hline Transtornos de ansiedade & 6 & 35 & 63 & 10,4 & 1 & 05 & 55 & 6,59 & $0,032^{*}$ \\
\hline Problemas Desenvolvimentais Invasivos & 6 & 35 & 60 & 8,57 & 1 & 05 & 53 & 4,96 & $0,011^{*}$ \\
\hline Transtorno de Déficit Atenção/Hiperatividade & 4 & 23 & 59 & 8,86 & 1 & 05 & 55 & 5,31 & 0,330 \\
\hline Transtorno Desafiador e opositor & 4 & 23 & 56 & 9,86 & 1 & 05 & 54 & 5,12 & 0,776 \\
\hline
\end{tabular}

Legenda: $f=$ freqüência; $\mathrm{DP}=$ desvio-padrão; ${ }^{*} p \leq 0,05$; teste de $\mathrm{t}$ de Student .

Tabela 3. Comparação dos indicadores cognitivos de pré-escolares nascidos prematuro e com baixo peso (G1) e a termo (G2)

\begin{tabular}{|c|c|c|c|c|c|c|}
\hline \multirow[b]{2}{*}{ Provas } & \multirow[t]{2}{*}{ Grupos } & \multicolumn{2}{|c|}{ G1-PTBP } & \multicolumn{2}{|c|}{ G2-AT } & \multirow{2}{*}{$\begin{array}{l}\text { G1 X G2 } \\
\text { ( } p \text {-valor) }\end{array}$} \\
\hline & & M & DP & M & DP & \\
\hline Columbia - Percentil & & 52,81 & 6,99 & 66,18 & 19,58 & $0,041^{*}$ \\
\hline Raven - Percentil & & 48,5 & 31 & 47,3 & 28,2 & 0,449 \\
\hline CATM - Fase de Manutenção & & 0,33 & 0,26 & 0,43 & 0,22 & 0,240 \\
\hline CATM - Operações cognitivas & & 0,42 & 0,26 & 0,62 & 0,14 & $0,011^{*}$ \\
\hline CATM - Comportamento & & 0,68 & 0,22 & 0,80 & 0,08 & $0,041^{*}$ \\
\hline CATM - Comportamento ACFS & & 28,59 & 8,90 & 32,06 & 5,66 & 0,186 \\
\hline
\end{tabular}

Legenda: M= média; $\mathrm{DP}=$ desvio-padrão; * $p$-valor $\leq 0,05$ indica significância pelo teste de $t$ de Student.

trou que não houve diferença significativa entre os grupos quanto ao raciocínio analógico-dedutivo, sendo a média de G1-PTBP maior do que de G2-AT.

Também na prova cognitiva assistida os grupos não diferiram significativamente, agora em relação à proporção de acerto total na fase de MAN (final) do CATM (Tabela 3). No entanto, observa-se que, durante a prova assistida, a frequência de operações cognitivas e de comportamentos facilitadores do aprendizado de ambos os grupos foi maior do que não-facilitadores; porém, a proporção média do G2-AT foi significativamente maior. Em relação aos comportamentos avaliados pela ACFS, não houve diferença significativa entre os grupos.

Com base na proporção de acerto total nas fases do CATM, obteve-se o perfil de desempenho cognitivo das crianças (Figura 1), em que mais da metade (10) das crianças do G2-AT e cinco crianças do G1-PTBP apresentaram-se como "ganhadoras". Este perfil indica ganhos sob condição de ajuda da examinadora para resolver o problema e a manutenção do aprendizado das estratégias para solução da tarefa, mesmo após a suspensão da ajuda. Ocorreu o contrário no G1-PTBP, em que mais da metade das crianças (12) apresentaram um perfil de desempenho "não-mantenedor". Este resultado indica que, enquanto as crianças do G2-AT melhoram o desempenho na avaliação assistida na fase de MAN em relação à fase SAJ, as crianças do G1-PTBP apresentam dificuldade de manter seu desempenho após a suspensão da ajuda.
O desempenho das crianças na avaliação assistida também foi analisado pela comparação das médias dos níveis de ajuda utilizados pelos grupos durante a fase de ASS do CATM (Tabela 4).

O nível de ajuda mais utilizado para G1-PTBP foi o nível 4 - Ajuda concreta (40\%), seguida pelo nível 3 - Feedback analítico (20\%). Para G2-AT, o nível mais utilizado foi o nível 1 - Repetição da instrução (32\%) seguido pelo nível 4 - Ajuda Concreta (25\%). Houve diferença significativa entre o nível de ajuda 2 -Instrução analítica, utilizado com maior freqüência no G2-AT (Tabela 4).

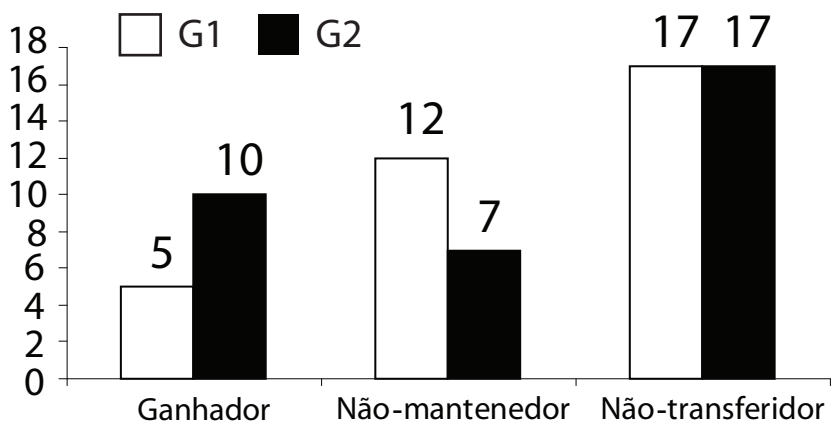

Figura 1. Frequência dos perfis de desempenho cognitivo de pré-escolares nascidos prematuros e com baixo peso (G1) e a termo (G2) na prova cognitiva assistida (CATM). 
Tabela 4. Comparação das médias dos níveis de ajuda utilizados para pré-escolares nascidos prematuros e com baixo peso (G1) e a termo (G2), durante a fase de assistência do CATM

\begin{tabular}{clccc}
\hline $\begin{array}{c}\text { Níveis de } \\
\text { ajuda }\end{array}$ & $\begin{array}{l}\text { Fase de } \\
\text { assistência }\end{array}$ & $\begin{array}{c}\text { G1 } \\
(\mathbf{n}=\mathbf{1 7})\end{array}$ & $\begin{array}{c}\text { G2 } \\
(\mathbf{n}=\mathbf{1 7})\end{array}$ & $\begin{array}{c}\text { G1 X G2 } \\
(\boldsymbol{p} \text {-valor })\end{array}$ \\
\hline 1 & Média & 0,32 & 0,40 & 0,2701 \\
& Desvio-padrão & 0,20 & 0,22 & \\
\hline 2 & Média & 0,09 & 0,20 & \multirow{2}{*}{$0,0195^{*}$} \\
& Desvio-padrão & 0,08 & 0,17 & \\
\hline \multirow{2}{*}{3} & Média & 0,20 & 0,13 & \multirow{2}{*}{0,0596} \\
& Desvio-padrão & 0,12 & 0,09 & \\
\hline \multirow{2}{*}{4} & Média & 0,40 & 0,25 & \multirow{2}{*}{0,0504} \\
& Desvio-padrão & 0,24 & 0,18 & \\
\hline
\end{tabular}

Legenda: * $p$-valor $\leq 0,05$ indica significância pelo teste de t de Student.

Resumindo, G1-PTBP diferenciou-se de G2-AT, apresentando pior desempenho em: (a) acertos totais do IAR, que mede habilidades básicas para a alfabetização; (b) capacidade de pronunciar palavras (vocabulário expressivo), pela LAVE; (c) problemas de comportamento, com maior freqüência principalmente em Transtornos de Ansiedade e Problemas Desenvolvimentais Invasivos, segundo os critérios de classificação do DSM-IV; (d) raciocínio geral, apesar de toda a amostra estar na média; (e) frequência de operações cognitivas facilitadoras na resolução das tarefas do CATM (f) frequência comportamentos facilitadores durante a prova assistida (g) níveis de ajuda - utilizando menos nível de ajuda 2 - Instrução analítica para resolução da tarefa.

\section{Discussão}

Dados comparativos da avaliação de 17 crianças nascidas pré-termo e com baixo peso (G1-PTBP) e 17 nascidas a termo (G2-AT), realizada aos cinco anos de idade, confirmaram estudos da área, mostrando diferenças estatisticamente significativas, desfavoráveis ao G1-PTBP, em relação aos indicadores acadêmicos, lingüísticos, comportamentais e cognitivos (Bohnert \& Breslau, 2008; Bhutha et al., 2002; Bordin et al., 2001; Feldman, 2007; Klebanov \& Brooks-Gunn, 2006; Linhares et al., 2006; Linhares et al., 2005; Martins et al., 2005; Méio et al., 2004; Pedromônico, 2006; Pereira \& Funayama, 2004). Entretanto, deve-se considerar o fato de ser uma amostra de conveniência, com mães do G1-PTBP dispostas a levar os filhos ao hospital para participar deste estudo, demonstrando interesse e preocupação com o desenvolvimento da criança, além da pesquisadora ter conhecimento prévio da identificação de cada criança, condições estas que restringem a generalização dos dados.

A prova avaliativa de habilidades básicas para a alfabetização - IAR (Leite, 1984) foi sensível para crianças de cinco anos de idade, permitindo identificar algumas habilidades e comportamentos básicos para a aprendizagem da leitura e da escrita, diferenciando G1-PTBP, que teve média inferior, principalmente em tarefas que envolvem habilidades não-verbais, como as noções de esquema corporal, posição, espaço, tamanho, quantidade, coordenação motora fina, discriminação visual e análise e síntese. Nesse sentido, essa prova acadêmica mostrou-se prescritiva, possibilitando uma análise descritiva das dificuldades dessas crianças, de forma a fornecer subsídios à elaboração de estratégias de ensino mais direcionadas às dificuldades encontradas nessa população e em momento anterior à entrada na escola. Pode, assim, atuar como meio de prevenção às possíveis dificuldades escolares dessas crianças.

A importância da avaliação e intervenção precoce na área da linguagem, nos casos de prematuridade e baixo peso (Pedromônico, 2006; Pereira \& Funayama, 2004), também foi levantada a partir dos resultados da avaliação do vocabulário expressivo, realizada pela LAVE (Capovilla \& Capovilla, 1997), uma vez que G1-PTBP apresentou uma média de palavras faladas significativamente abaixo da esperada para a idade de cinco anos, demonstrando dificuldades no repertório verbal em relação ao G2-AT, cujo desempenho, por sua vez, foi equivalente às crianças dessa idade. Essa dificuldade na linguagem expressiva pode se somar àquelas identificadas pelo IAR (Leite, 1984), contribuindo para futuros problemas de alfabetização.

Os cuidados com essas crianças do G1-PTBP foram também indicados na área cognitiva, pois este grupo apresentou dados significativamente menores em comparação ao G2-AT, coerentemente com os dados da área (Bhutha et al., 2002; Klebanov \& Brooks-Gunn, 2006; Linhares et al., 2005; Martins et al., 2005; Méio et al., 2004, entre outros), apesar de ambos os grupos não terem apresentado dificuldades quanto ao raciocínio geral quando comparados aos pares da mesma idade.

Esse perfil do G1-PTBP se repetiu nos dados da outra prova cognitiva usada - agora em formato assistido, ou seja, com ajuda da examinadora durante a execução da prova (Haywood \& Lidz, 2007; Linhares, Escolano \& Emuno, 2006) - em que a média de acerto total na fase final da prova não foi maior do que $50 \%$ para a maioria das crianças, significando um perfil cognitivo não-mantenedor. Em contrapartida, G2-AT apresentou média de acerto total indicativa de um perfil cognitivo de ganhador, melhorando o desempenho mesmo após a suspensão da ajuda. Complementando esses dados, a avaliação assistida permitiu identificar operações cognitivas e comportamentos facilitadores do desempenho cognitivo, os quais claramente diferenciaram as crianças PTBP, que se mostraram mais dispersivas, impulsivas, com dificuldade de generalização e de identificação de relevância, por exemplo, contribuindo, assim, para as dificuldades no estabelecimento e na manutenção dos ganhos de desempenho alcançados sob a condição de assistência na prova assistida. De outro lado, esses dados devem ser vistos com cuidado, pois, na idade pré-escolar, as operações cognitivas podem estar ainda em processo de desenvolvimento, sendo, por isso, esperado um perfil mais desatento, impulsivo e com menor autorregulação do comportamento (Tzuriel, 2001). No entanto, as crianças do G2-AT demonstraram mais acessibilidade à mediação e padrões cognitivos mais facilitadores, como refletir e analisar os estímulos antes de dar a resposta, identificar alternativas de respostas e analisá-las comparativamente, e distinguir dados relevantes dos 
irrelevantes em um problema analógico. Este padrão pode ter sido o grande responsável pela diferença de desempenho entre os grupos, em consonância com o desempenho dessas crianças do G1-PTBP no trabalho de Turrini et al., (2010), também indicando a importância de intervenções educativas, com foco no manejo da regulação do comportamento, conforme analisam Olson et al. (2009).

$\mathrm{O}$ uso de avaliação assistida gerou um diferencial deste estudo, na medida em que contribuiu para compreender melhor os padrões cognitivos dessas crianças prematuras e com baixo peso. Essa informação pode subsidiar o planejamento de estratégias de intervenção mais detalhadas e direcionadas às dificuldades encontradas (Linhares et al., 2006).

Para além desses dados obtidos e considerando que o processo de escolarização infantil não depende somente das habilidades lingüísticas e cognitivas da criança, mas também de seus comportamentos afetivo-motivacionais, a identificação e intervenção nos casos de problemas de comportamento tornam-se relevantes na prevenção de problemas escolares, especialmente para essa população estudada (Olson et al., 2009; Turrini et al., 2010; Tzuriel, 2001). Assim devem ser considerados os dados obtidos pelo CBCL (1 1 $1 \frac{2-5}{2}$ anos) (Achenbach \& Rescorla, 2004), mostrando diferenças significativas entre os grupos em termos de Transtornos Mentais, segundo o DSM-IV, especialmente nas Escalas de Transtorno de Ansiedade (preocupação excessiva, associada à inquietação, fadiga excessiva, dificuldade de concentração, irritabilidade) e de Transtorno Desenvolvimental Invasivo (comprometimento nas habilidades de interação social, de comunicação, e nos interesses e atitudes das crianças). Esses dados são também indicativos da necessidade de encaminhamento para acompanhamento psicológico ou psiquiátrico para as crianças do G1-PTBP, confirmando pesquisas da área (Bohnert \& Breslau, 2008; Bordin et al., 2001; Linhares et al., 2005).

Os resultados deste estudo, que diferenciaram significativa e negativamente as crianças do G1-PTBP, aos cinco anos de idade, nas áreas acadêmicas, de linguagem, cognitiva e comportamental, também permitiram identificar a presença de recursos nessas crianças e sinais de resiliência (vide o desempenho cognitivo superior no Raven-MPC). Diante disso, fica claro que a relação da prematuridade e do baixo peso ao nascimento com o comprometimento no desenvolvimento não pode ser entendida como uma relação direta de causa-e-efeito mas, ao contrário, demonstra a necessidade de se identificar os mecanismos protetores, capazes de minimizar e até neutralizar os efeitos potenciais do risco ao desenvolvimento (Bordin et al., 2001; Linhares et al., 2005; Linhares et al., 2006; Luthar et al., 2006). Sendo assim, este estudo alinha-se àqueles que avaliam a interação entre os fatores de risco biológico, social e ambiental, podendo influenciar, seja de forma positiva ou negativa, a trajetória de desenvolvimento da criança.

Espera-se que os resultados encontrados possam ajudar os profissionais da Psicologia e da Educação a compreender a criança em sua complexidade, fornecendo subsídios teóricos e metodológicos que possibilite o planejamento de atividades mais direcionadas às necessidades de crianças em risco potencial para problemas de desenvolvimento.

\section{Referências}

Achenbach, T. M., \& Rescorla, L. A. (2004). Child Behavior Checklist for Age 11/2-5 (CBCL/11/2-5/LDS). Mental health practitioners ' guide for the Achenbach System of Empirically Based Assessment (ASEBA) (M. R. Santa-Maria Menguel \& M. B. M. Linhares, Trad.). Burlington, VT: University of Vermont, Research Center for Children, Youth \& Families. Trabalho não publicado.

Angelini, A. L., Alves, I. C. B., Custódio, E. M., Duarte, W. F., \& Duarte, J. L. M. (1999). Manual Matrizes Progressivas Coloridas de Raven: Escala especial. São Paulo: Centro Editor de Testes e Pesquisas em Psicologia.

Associação Brasileira de Empresas de Pesquisa - ABEP (2008). Critério de Classificação Econômica Brasil. Retirado em 03/10/2008, de http://www.abep.org/?usaritem= arquivos\&iditem $=23$.

Bhutta, A. T., Cleves, M. A., Casey, P. H., Cradocl, M. M., \& Anand, K. J. S. (2002). Cognitive and behavioral outcomes of school-aged children who were born preterm: A meta-analysis. Journal of the American Medical Association, 288, 728-737.

Bohnert, K. M., \& Breslau, N. (2008). Stability of psychiatric outcomes of low birth weight: A longitudinal investigation. Archives of General Psychiatry, 65(9), 1080-1086.

Bordin, M. B. M., Linhares, M. B. M., \& Jorge, S. M. (2001). Aspectos cognitivos e comportamentais na média meninice de crianças nascidas pré-termo e com muito baixo peso. Psicologia: Teoria e Pesquisa, 17, 49-57.

Bordin, I. A. S., Mari, J. J., \& Caiero, M F. (1995). Validação da versão brasileira do Child Behavior Checklist (CBCL): Inventário de comportamentos da infância e adolescência: Dados preliminares. Revista ABP APPAL, 17(2), 55-66.

Burgmeister, B. B., Blum, L. H., \& Lorge, I. (2001). Escala de Maturidade Mental Columbia: Manual para aplicação e interpretação (I. C. B. Alves \& J. L. M. Duarte, Trad., padronização brasileira). São Paulo: Casa do Psicólogo.

Capovilla, F. C., \& Capovilla, A. G. S. (1997). Desenvolvimento lingüístico na criança dos dois aos seis anos: Tradução e estandardização do Peabody Picture Vocabulary Test de Dunn \& Dunn, e da Language Development Survey de Rescorla. Ciência Cognitiva: Teoria Pesquisa e Aplicação, 1(1), 353-380.

Carretoni Filho, H., \& Prebianchi, H. B. (1994). Exame clínico psicológico (Anamnese). Campinas: Editorial Psy.

Enumo, S. R. F (2005). Avaliação Assistida para crianças com necessidades educativas especiais: um recurso auxiliar na inclusão escolar. Revista Brasileira de Educação Especial, 11(3), 335-354.

Fagundes, A. J. F. M. (1982). Descrição, definição e registro do comportamento. São Paulo: Edicon.

Feldman, R. (2007). Maternal versus child risk and the development of parent-child and family relationships in five high-risk populations. Development and Psychopathology, 19, 293-312.

Gauy, F. V., \& Guimarães, S. S. (2006). Triagem em saúde mental infantil. Psicologia: Teoria e Pesquisa, 22, 5-16.

Haywood, H. C., \& Lidz, C. S. (2007). Dynamic assessment in practice: Clinical and educational applications. New York. Cambridge University Press.

Klebanov, P., \& Brooks-Gunn, J. (2006). Cumulative, human capital, and psychological risk in the context of early intervention: Links with IQ at ages 3, 5, and 8. Annals of New York Academy of Sciences, 1094, 63-82. 
Leite, S. A. (1984). Instrumento de avaliação de repertório básico para a alfabetização (IAR). São Paulo: Edicon.

Linhares, M. B. M., Carvalho, A. E. V., Correia, L. L., Gaspardo, C. M., \& Padovani, F. H. P. (2006). Psicologia pediátrica e neonatologia de alto risco: Promoção precoce do desenvolvimento de bebês prematuros. In M. A. Crepaldi, M. B. M. Linhares \& G. B. Perosa (Eds.), Temas em Psicologia Pediátrica (pp. 39-74). São Paulo: Casa do Psicólogo.

Linhares, M. B. M., Chimello, J. T., Bordin, M. B. M., Carvalho A. V. E., \& Martinez, F. E. (2005). Desenvolvimento psicológico na fase escolar de crianças nascidas pré-termo em comparação a crianças nascidas a termo. Psicologia: Reflexão e Crítica, 18(1), 119-127.

Linhares, M. B. M., Escolano, A. C. M., \& Enumo, S. R. F. (2006). Avaliação cognitiva assistida: Fundamentos teórico-conceituais e contribuições. In M. B. M. Linhares, A. C. M. Escolano \& S. R. F. Enumo (Eds.), Avaliação assistida: Fundamentos, Procedimentos e aplicabilidade (pp. 16-32). São Paulo: Casa do Psicólogo.

Linhares, M. B. M., Santa Maria, M. R., \& Escolano, A. C. (2006). Indicadores de desempenho cognitivo na resolução dos problemas de busca-Tipos de perguntas e de soluções. In M. B. M. Linhares, A. C. M. Escolano \& S. R. F. Enumo (Eds.), Avaliação assistida: Fundamentos, Procedimentos e aplicabilidade (pp. 16-32). São Paulo: Casa do Psicólogo.

Luthar, S. S., Sawyer, J. A., \& Brown, P. (2006). Conceptual issues in studies of resilience: Past, present, and future research. Annals of New York Academy of Sciences, 1094, 105-115.

Martins, I. M. B; Linhares, M. B. M., \& Martinez, F. E (2005). Indicadores de desenvolvimento na fase pré-escolar de crianças nascidas pré-termo. Psicologia em Estudo, 10(2), 235-243.

Méio, M. D. B. B., Lopes, C. S., Morsch, D. S., Monteiro, A. P. G., Rocha, S. B., Borges, R. A., \& Reis, A. B. (2004). Desenvolvimento cognitivo de crianças prematuras de muito baixo peso na idade pré-escolar. Jornal de Pediatria, 80, 495-502.

Olson, S. L., Sameroff, A. J., Lukenheimer, E. S., \& Kerr, D. C. (2009). Self-regulatory processes in development of disruptive behavior problems: The preschool-to-school transition. In S. L. Olson \& A. J. Samerof (Eds.), Byopsychosocial regulatory process in the development of childhood behavioral problems (pp. 144-185). New York: Cambridge University Press.
Pedromônico, M. R. M. (2006). A relevância da avaliação psicológica na clínica pediátrica. In M. A. Crepaldi, M. B. M. Linhares \& G. B. Perosa (Eds..), Temas em Psicologia Pediátrica (pp. 83-107). São Paulo: Casa do Psicólogo.

Pereira, M. R., \& Funayama, C. A. R. (2004). Avaliação de alguns aspectos da aquisição e desenvolvimento da linguagem de crianças nascidas pré-termo. Arquivos de Neuropsiquiatria. 62(3-A), 641-648.

Pestana, M., \& Gageiro, J. (2003). Análise dos dados para Ciências Sociais: A complementaridade do SPSS. Lisboa: Edições Silaba.

Rugolo, L. M. S. S. (2005). Crescimento e desenvolvimento a longo prazo do prematuro extremo. Jornal de Pediatria, 81(1), 101-110.

Santa Maria, M. R., \& Linhares, M. B. M. (1999). Avaliação cognitiva assistida de crianças com indicações de dificuldade de aprendizagem escolar e deficiência mental leve. Psicologia: Reflexão e Crítica, 12(2), 395-417.

Turrini, F. A., Enumo, S. R. F., Ferrão, E. S., \& Monteiro, R. N. (2010). Comportamentos afetivo-motivacionais durante prova assistida diferenciam pré-escolares nascidos prematuros e com baixo peso dos nascidos a termo. Psicologia: Teoria e Prática, $12(2), 158-172$.

Tzuriel, D. (2001). Dynamic assessment of young children. New York: Kluwer Academic/ Plenum Publishers.

Tzuriel, D., \& Klein, P. S. (1990). The Children's Analogical Thinking Modifiability Test: Instruction manual. Ramat-Gan: School of Education Bar Ilan University.

Recebido em 03.04.2009

Primeira decisão editorial em 05.01.2011

Versão final em 28.06.2011

Aceito em 18.07.2011 